DOI: $10.217672576-3903.100002$

\title{
Philadelphia-Positive Acute Myeloblastic Leukemia: A Rare Entity
}

\author{
Smeeta Gajendra ${ }^{1}$ and Sahoo MK ${ }^{2}$ \\ ${ }^{1}$ Department of Pathology and Laboratory Medicine, Gurgaon, Haryana, India \\ ${ }^{2}$ Department of Nuclear Medicine, All India Institute of Medical Science, New Delhi, India
}

Corresponding author: Smeeta Gajendra, Associate Consultant, Department of Pathology and Laboratory Medicine, Medanta-The Medicity, Sector - 38, Gurgaon, Haryana 122 001, India, Tel: 09013590875, Fax: +91- 1244834 111; E-mail: drsmeeta@gmail.com

Received date: March 13, 2016; Accepted date: April 06, 2016; Published date: April 12, 2016

Citation: Gajendra S, Sahoo MK. Philadelphia-Positive Acute Myeloblastic Leukemia: A Rare Entity. J Neoplasm 2016, 1:1

\section{Definition}

Philadelphia positive $(\mathrm{Ph}+)$ Acute Myeloblastic Leukemia $(A M L)$ is an acute onset neoplasm of myeloblasts in which the blasts harbor BCR/ABL translocation in the absence of a clinical history of chronic phase or accelerated phase chronic myeloid leukemia (CML) and a lack of clinical and laboratory features of $\mathrm{CML}$, such as splenomegaly and basophilia.

Keywords: Philadelphia chromosome; BCR/ABL; Acute myeloid leukemia

\section{Genetics}

The Philadelphia chromosome was first discovered in Philadelphia in 1960. It corresponds to shortened chromosome 22 resulting from reciprocal translocation between long arm of chromosome 9 and short arm of chromosome 22 , which is designated as $t(9 ; 22)(q 34 ; q 11)$. This gives rise to a $B C R / A B L$ fusion gene, that juxtaposes the $A B L 1$ gene on chromosome 9 (region q34) to a part of the $B C R$ ("Breakpoint Cluster Region") gene on chromosome 22 (region q11). There are two major forms of the BCR/ABL fusion gene of different sizes involving $A B L$ exon 2 with different exons of $B C R$ gene. Major $B C R(M-B C R)$ resulting in $b 2 a 2$ and $b 3 a 2$ mRNA transcripts, p210 chimeric protein, are most commonly involved in CML patients. Minor BCR (m-BCR), e1a2 mRNA transcripts and p190 chimeric protein, are most frequently associated with $\mathrm{Ph}$ positive Acute lymphoblastic leukemia $(A L L)$ and in many patients with CML. Third less common fusion gene involving breakpoint in micro BCR ( $\mu$-BCR), e19a2 and result in p230 chimeric protein, may demonstrate prominent neutrophilic leukocytosis; these should be diagnosed as chronic neutrophilic leukaemia [1,2].

\section{Mechanism}

The $A B L$ gene expresses a membrane associated protein, a tyrosine kinase, and the $B C R-A B L$ transcript is also translated into a tyrosine kinase. The mutant tyrosine kinase of the BCR$A B L$ transcript codes for a protein that is continuously activated and subsequently activates a number of cell cycle controlling proteins and enzymes resulting in unregulated cell division. Several functional domains have been identified in the $B C R-A B L$ protein that may contribute to cellular transformation. In the ABL portion, these domains are the $\mathrm{SH} 1$ (tyrosine kinase), $\mathrm{SH} 2$ and actin-binding domains and in the BCR portion, they are the coiled-coil oligomerization domain comprised between amino acids (aa) 1-63, the tyrosine at position 177 (GRB-2 binding site) and the phosphor serine/ threonine rich $\mathrm{SH} 2$ binding domain. The increased tyrosine kinase activity of $B C R-A B L$ fusion protein results in phosphorylation of several cellular substrates and autophosphorylation of BCR-ABL, which in turn induces recruitment and binding of a number of adaptor molecules and proteins. BCR-ABL activates signal transduction pathways such as RAS/MAPK, PI-3 kinase, c-CBL and CRKL pathways, JAKSTAT and the SRC pathway. The major role in transformation and proliferation is played by the RAS, JUN-kinase, and PI-3 kinase pathways. Inhibition of apoptosis is thought to result from activation of the PI-3 kinase and RAS pathways, with induction through AKT of C-MYC and BCL-2. Unregulated cell proliferation with inhibition of apoptosis pays a major role in oncogenesis in Ph+ leukemias [3,4].

\section{Epidemiology}

The p210 fusion protein is characteristic of CML, occurring in about $95 \%$ of the cases and in $20 \%$ of $\mathrm{Ph}+\mathrm{ALL}$, in which the p190 fusion protein is more frequent. The p230 is usually associated with chronic neutrophilic leukemia. The incidence of Philadelphia chromosome in $\mathrm{Ph}+\mathrm{AML}$ is approximately $0.5-3 \%$ as reported by various groups [2]. An incidence of $<1 \%$ was reported in the studies where the biphenotypic and bilineal AML were excluded and only pure de novo cases of AML were considered. A higher incidence of $3 \%$ was reported when biphenotypic or bilineal cases were included in the studies [5]. Most of the $\mathrm{Ph}+\mathrm{AML}$ cases expressed the p210 $B C R-A B L$ transcript which is typical of CML-MBC and few Ph+ AML cases expressed a p190 BCR-ABL transcript, which is only rarely expressed in CML-MBC.

\section{Clinical Features}

Clinical presentations are similar to that of acute myeloid leukemia. However, $\mathrm{Ph}$ positivity in $\mathrm{AML}$ is more common in adult than children. 


\section{Diagnosis of $\mathrm{Ph}$ chromosome (BCR/ABL translocation)}

Cytogenetic and molecular analysis plays important role in the diagnosis and monitoring of the disease to assess complete cytogenetic and molecular response. Peripheral blood and bone marrow samples are examined for $\mathrm{Ph}$ chromosome. Conventional $\mathrm{G}$ banding karyotyping is the most simple and cost-effective technique and can be able to demonstrate $\mathrm{Ph}$ chromosome in approximately $95 \%$ of cases; however, rest of the cases require more sensitive molecular methods. In conventional karyotyping, Ph chromosome positivity is expressed as percentage of $\mathrm{Ph}+$ metaphases out of all metaphases analyzed. A minimum of 20 metaphases were analyzed routinely according to ISCN guidelines. Fluorescence in situ hybridization (FISH) can be performed on metaphase spreads and interphase nuclei, using locus specific probes for $B C R-A B L$ gene. In normal interphase nuclei four separate fluorescent signals i.e., two green and two red are seen. In $\mathrm{Ph}$ +ve nuclei, however, there are two separate signals which represent the two normal chromosomes and an orange-green doublet or a yellow signal corresponding to BCR-ABL fusion gene on chromosome 22. The more advance molecular technique as reverse transcriptase polymerase chain reaction (RT-PCR), total RNA was extracted and is reverse transcribed to make complimentary DNA (CDNA). PCR primers specific to the DNA sequences of portions of BCR/ABL were used to amplify the CDNA. The PCR products were separated by agarose gel electrophoresis and visualized by UV transillumination. The real time PCR (RQ-PCR) assay detects e1a2, e13a2, and e14a2 transcripts in a single tube and is normalized to $A B L 1$, with BCR-ABL transcript type(s) determined by subsequent capillary electrophoretic separation of the fluorochrome labeled products. This RQ-PCR can detect residual disease in a very low level [5].

\section{Morphology, cytochemistry and immune- phenotyping}

There is no difference in morphology, cytochemistry and immunophenotyping features that differentiate it from other type of AML.

\section{Differential diagnoses}

The most important differential diagnosis of $\mathrm{Ph}+\mathrm{AML}$ is CML in myeloid blast crisis (CML-MBC). The criteria suggested to differentiate $\mathrm{Ph}+\mathrm{AML}$ from $\mathrm{CML}-\mathrm{MBC}$ include: acute presentation with a short history, lack of history of previous chronic phase and also lack splenomegaly. The age of presentation of ph+ AML is earlier in comparison with CML$M B C$. It also lacks peripheral blood and bone marrow basophilia and also has a lower peripheral blood maturing myeloid cell count (excluding blasts) at presentation than the $\mathrm{CML}-\mathrm{MBC}$ group. The $\mathrm{Ph}+\mathrm{AML}$ has lower bone marrow cellularity and myeloid/erythroid ratio. Presence of dwarf megakaryocytes is common in CML-BC which is a rare finding in $\mathrm{Ph}+\mathrm{AML}$. There is no significant difference in total leucocyte count, percentage of eosinophil's, or monocytes. There was also no difference in the number of megakaryocytes or in erythroid, myeloid, or megakaryocyte dysplasia [6-8]. There is also no difference in percentage and immune-phenotyping of blasts between the two groups. Cytogenetic and molecular features can be useful in distinguishing this pathology. In CML$\mathrm{MBC}$, clonal evolution, which is defined as additional chromosomal abnormalities, occurs in $60 \%$ to $80 \%$. The most common additional abnormalities are extra $\mathrm{Ph}$ chromosome, trisomy 8, trisomy 19 or isochromosome 17q. However, an additional chromosomal abnormality in $\mathrm{Ph}+\mathrm{AML}$ occurs in $25 \%$ to $55 \%$ cases. Few studies Deletion $7 q(-7 q)$ abnormalities were present in case of $\mathrm{Ph}$ positive $\mathrm{AML}$, as demonstated by few series. Association of mutations suppressing cell differentiation (Core Binding Factor $A M L$ ) and mutations increasing cell proliferation (as FLT3 gene, KIT gene) is more frequent in $\mathrm{Ph}+\mathrm{AML}$ than in CML-MBC. Two recent studies have shown that Nucleophosmin (NPM1) is commonly mutated in $A M L$ and absent in CML-MBC, whereas $A B L 1$ mutations are common in $\mathrm{CML}[9,10]$. Another differential diagnosis is mixed phenotypic acute leukemia with $t(9 ; 22)$ (q34;q11.2) which can be differentiated by having dimorphic blast population as demonstrated by immune-phenotyping [myeloid as by myeloperoxidase or monocytic differentiation (any 2 of CD14, CD64, CD11c, NSE, lysozyme) with B lymphoid (CD19 with 1 of CD79a, CD10, CD22) or T lymphoid (surface) cytoplasmic CD3)] [11].

\section{Treatment}

This disease shows resistance to conventional chemotherapy protocols. Imatinib mesylate is a selective BCR$A B L$ tyrosine kinase inhibitor, has shown significant antileukemic activity in patients with $\mathrm{Ph}+$ leukemia. Imatinib as monotherapy failed to show efficacy as induction therapy. However, when Imatinib is started after achieving complete response with AML chemotherapy, it allows in achieving a complete cytogenetic and molecular response. The doses of Imatinib reported in the literature are $400 \mathrm{mg}$ and $600 \mathrm{mg} /$ day. Some authors suggested as the patients are at considerable risk of meningeal leukemia during monotherapy, they should routinely receive CNS prophylaxis, cranial irradiation or intrathecal chemotherapy [12]. A combination of AML chemotherapy with maintenance of Imatinib can provides remission and a subsequent allogeneic stem cell transplant (ASCT) is the best consolidation therapy for the patients with $\mathrm{Ph}+\mathrm{AML}$ [3]. The median and the overall survival of the patients can be increased by addition of Imatinib to the conventional AML therapy followed by ASCT.

\section{Prognosis}

Induction failure and relapse is also common in $\mathrm{Ph}+\mathrm{AML}$. Long-term survival may be achieved with HSCT as earlier as after complete remission from Imatinib and chemotherapy treatmentanalyzed $12 \mathrm{Ph}+\mathrm{AML}$ patients and showed a median overall survival $24(8-80)$ months and the the overall survival of 3 years was (51.4 $\pm 17.7 \%)$ [13]. 


\section{Conclusion}

The $\mathrm{Ph}+\mathrm{AML}$ is a distinct clinico-pathological entity and not a de novo presentation of CML-myeloid blast crisis. The most recent version of the World Health Organization (WHO) classification does not include AML with t $(9 ; 22)(q 34 ; q 11.2) /$ BCR-ABL1 Ph+ as an different entity. But it should be differentiates from CML-MBC as both have different clinical, morphological, cytogenetic and molecular features. A combination of AML chemotherapy followed by maintenance by Imatinib can provides complete cytogenetic and molecular response and ASCT can further provides a long term survival.

\section{References}

1. Arana-Trejo RM, Ruíz Sánchez E, Ignacio-lbarra G, Báez de la Fuente E, Garces O, et al. (2002) BCR/ABL p210, p190 and p230 fusion genes in 250 Mexican patients with chronic myeloid leukaemia (CML). Clin Lab Haematol 24: 145-150.

2. Keung YK, Beaty M, Powell BL, Molnar I, Buss D, et al. (2004) Philadelphia chromosome positive myelodysplastic syndrome and acute myeloid leukemia- retrospective study and review of literature. Leuk Res 28: 579-86.

3. Salesse $S$, Verfaillie CM (2002) BCR/ABL: from molecular mechanisms of leukemia induction to treatment of chronic myelogenous leukemia. Oncogene. 21: 8547-8559.

4. Jones D, Luthra R, Cortes J, Thomas D, O'Brien S, et al. (2008) $B C R-A B L$ fusion transcript types and levels and their interaction with secondary genetic changes in determining the phenotype of Philadelphia chromosome-positive leukemias. Blood 112: 5190-5192.

5. Luthra R, Medeiros LJ (2006) Taq Man reverse transcriptase polymerase chain reaction coupled with capillary electrophoresis for quantification and identification of BCR-ABL transcript type. Methods Mol Biol 335: 135-145.
6. Soupir CP, Vergilio JA, Dal CP, Muzikansky A, Kantarjian $\mathrm{H}$, et al. (2007) Philadelphia chromosome-positive acute myeloid leukemia: a rare aggressive leukemia with clinicopathologic features distinct from chronic myeloid leukemia in myeloid blast crisis. Am J Clin Pathol 127: 642-650.

7. Konoplev S, Yin CC, Kornblau SM, Kantarjian HM, Konopleva M, et al. (2013) Molecular characterization of de novo Philadelphia chromosome-positive acute myeloid leukemia. Leuk Lymphoma 54: $138-144$

8. Frankfurt O, Platanias LC (2013) Philadelphia chromosome positive acute myeloid leukemia or de novo chronic myeloid leukemia-blast phase? Leuk Lymphoma 54: 1-2.

9. Jameel A (2012) Leukemia: Philadelphia chromosome positive acute myeloid a rare hematological disorder: A case report. J Postgrad Med Inst 26: 111-114.

10. Reboursiere E, Chantepie S, Gac AC, Reman O (2015) Rare but authentic Philadelphia-positive acute myeloblastic leukemia: two case reports and a literature review of characteristics, treatment and outcome. Hematol Oncol Stem Cell Ther 8: 28-33.

11. Borowitz $M$, Bene $M C$, Harris NL, Porwit A, Matutes $E$, et al. (2008) Acute leukemias of ambiguous lineage. World Health Organization Classification of Tumours: Pathology and Genetics of Tumours of Haematopoietic and Lymphoid Tissues. IARC Press, Lyon, France.

12. Amor IB, Mseddi SH, Kassar O, Mnif S, Jdidi I, et al. (2013) Philadelphie chromosome-positive de novo acute myeloid leukemia. Isolated meningeal relapse in a patient treated with imatinib mesylate. Case Reports in Clinical Medicine 2: 167-169.

13. Fei $X H$, Wu SL, Sun RJ, Zhou JR, Wang JB, et al. (2012) Clinical analysis of 12 cases of acute myeloid leukemia with $\mathrm{Ph}$ chromosome and BCR-ABL positive. Zhongguo Shi Yan Xue Ye Xue Za Zhi 20: 545-548. 